\title{
Designing a Genetic Algorithm for Efficient Calculation in Time-Lapse Gravity Inversion
}

\author{
Eko Januari Wahyudi, Djoko Santoso, Wawan Gunawan Abdul Kadir \& \\ Susanti Alawiyah \\ Applied Geophysics Research Group, Faculty of Mining and Petroleum Engineering, \\ Institut Teknologi Bandung, Jl. Ganesha 10 Gd. BSC-B Lt.2, Bandung 40132, Indonesia \\ Email: ekojw@gf.itb.ac.id
}

\begin{abstract}
As an advanced application of soft computation in the oil and gas industry, genetic algorithms (GA) can contribute to geophysical inversion problems in order to achieve better results and efficiency in the computational process. Time-lapse gravity responses to pore-fluid density changes can be modeled to provide the density distribution in the subsurface. This paper discusses the progress of work in inverse modeling of time-lapse gravity data using value encoding with alphabet formulation. The alphabet formulation was designed to provide the solution for positive and negative density change with respect to a reference value $(0 \mathrm{gr} / \mathrm{cc})$. The inversion was computed using a genetic algorithm as the optimization method. Working with genetic algorithms, time-intensive computational processes are a challenge, so the algorithm was designed in steps through the evaluation of a GA operator performance test. The performances of several combinations of GA operators (selection, crossover, mutation, and replacement) were tested with a synthetic model of a single-layer reservoir. Sharp boundaries of density changes in the reservoir layer were derived from interpretation of the averaged calculation of several model samples. Analysis showed that the combination of stochastic universal sample-multipoint crossover-quenched simulated annealing per generation-no duplicity achieved the most promising results.
\end{abstract}

Keywords: genetic algorithm; inverse modeling; optimization; reservoir monitoring; time-lapse gravity.

\section{$1 \quad$ Introduction}

In recent years, the development of gravity inversion has been dominated by two methodologies: interface inversion and generalized density inversion. In time-lapse gravity inverse problems, computation of the interface inversion seeks a solution for the boundaries that separate the discrete density contrast layers in the subsurface (such as ground water lowering [1,2] or the flow front of injected water in enhanced oil recovery (EOR)), while computation of the generalized density inversion can provide the distribution of the density anomalies as a space function [3].

Received April 29 $9^{\text {th }}, 2013$, Revised October $25^{\text {th }}, 2013$, Accepted for publication January $18^{\text {th }}, 2014$.

Copyright (C) 2014 Published by ITB Journal Publisher, ISSN: 2337-5779, DOI: 10.5614/j.eng.technol.sci.2014.46.1.4 
A benefit of the interface inversion method is that it allows the user to input the density change from pore-fluid replacement directly. For example, the bulk density change from fluid replacement in the reservoir's porosity caused by water injection can be estimated with a simple calculation using available/ assumed reservoir data. In the interface inversion, a well-defined input density is directly utilized to seek a solution for the water-hydrocarbon boundary in certain time steps as injected water sweeps into the reservoir. The generalized density inversion, on the other hand, has the flexibility to handle complex anomalies. The solution for the generalized density inversion is relatively easy to compute because of the linear relationship between observation data and density contrast.

A limitation of the interface inversion method is the assumption of a simple topology that will show an effect if non-target anomalies from shallow sources are not succesfully removed during data processing. A miss-match between the assumed model and the data can lead to large errors and even failure of the inversion. In addition, the problem is not linear and can be more difficult to compute. A difficulty that arises from the generalized density inversion method is that calculation with continuous values of model parameters will produce intermediate density. Even implementation of density bounds does not succesfully produce strong constraints in the inversion solution. The data are satisfied with an intermediate density distribution and only recover a portion of the causative body.

To overcome the difficulties associated with both methods, several authors have used a binary formulation [4-7]. For wide implementation of a binary inversion, the model parameters used in the calculation are set to discrete terms. For timelapse gravity problems, the values of the discrete density changes are defined by involving consideration of dynamic processes in the reservoir (for example fluid contact movements). The binary method accommodates subsurface conditions by setting suitable model parameter values and the solution is expected to gain sharp boundaries between the discrete density values. Implementation of a binary formulation with a discrete nature makes the derivative-based minimization technique no longer applicable. This is because a highly constrained inversion has limited options in solution variables and therefore this kind of inversion needs an optimization algorithm.

Krahenbuhl \& Li [4-7] utilized the genetic algorithm (GA) approach to solve the optimization of their binary gravity inversion. The appeal of applying a GA to optimization problems is that one can expect to gain wider results in a geophysical inversion. The main obstacle when using a GA is the computational cost. A GA alone as optimization algorithm usually needs ample execution time (ET) to explore the best solution. The necessity of more efficient computation 
can be met with by using a hybrid optimization method. Krahenbuhl \& Li [5] combined a GA with quenched simulated annealing (QSA). They demonstrated a time-lapse gravity inversion to characterize two values of discrete density, for example: 0.00 and $0.35 \mathrm{gr} / \mathrm{cc}$. This kind of binary formulation has the limitation that it can only characterize one single density change with respect to the reference value $(0.00 \mathrm{gr} / \mathrm{cc})$, whereas several studies in time-lapse gravity anomalies [8-12] show at least two types of density change characterization in reservoir layers. You need a sufficient formulation to elaborate real conditions with a time-lapse gravity inversion. Two types of density change characterization with respect to the reference value are: positive density change $(+\Delta \rho)$ and negative density change $(-\Delta \rho)$.

In this work, we have developed a time-lapse gravity inverse modeling method using three density change values $(+\Delta \rho, 0$, and $-\Delta \rho)$. The inversion calculation utilizes alphabet formulation as constraint for the model parameters. Previous studies by Wahyudi [13,14] and Wahyudi, et al. [15]-[17] have shown that time-lapse gravity inversion to characterize positive and negative density change with a 2-bit binary GA formulation is still inefficient because of the redundant number of discrete model parameters. In this paper, the design of a GA through the evaluation of a GA operator performance test is discussed. The performances of several combinations of GA operators were tested with a synthetic model of a single-layer reservoir to seek the most efficient combination of GA operators for the time-lapse gravity problem.

\section{Inversion Methodology}

In this section, we describe the inversion of time-lapse gravity data from a single-layer reservoir using alphabet formulation. The solution results are restricted to three values of the discrete model parameters (positive density change, zero, and negative density change). The algorithm in this study was designed to accommodate constrained density values derived from pore-fluid replacement occurring in the reservoir caused by injection and production activities. This kind of formulation reduces the interpreter's subjectivity, especially in the stage of density change characterization.

Compared with a binary formulation, value encoding with alphabet formulation $[\mathrm{A}, \mathrm{B}, \mathrm{C}]$ elaborates more options for the discrete model parameters. Each cell in the reservoir grid has only three possible model parameters. The alphabet formulation in the time-lapse gravity inversion was implemented as follows:

1. The zone of positive density change was defined as group A,

2. The zone of reference (zero density change) was defined as group B, and

3. The zone of negative density change was defined as group $\mathrm{C}$. 
The optimization algorithm seeks the minimum objective function $(\varphi)$, which consists of the total model objective function $\left(\varphi_{\mathrm{m}}\right)$ with a trade-off parameter $(\lambda)$ and a data misfit function $\left(\varphi_{\mathrm{d}}\right)$. The objective function was defined as:

$$
\varphi=\varphi_{\mathrm{d}}(\rho)+\lambda \varphi_{\mathrm{m}}(\tau), \text { with } \rho \in\{\mathrm{A}, \mathrm{B}, \mathrm{C}\} .
$$

The data misfit function $\left(\varphi_{\mathrm{d}}\right)$ is defined by Krahenbuhl [18] as:

$$
\varphi_{\mathrm{d}}=\sum_{\mathrm{i}=1}^{\mathrm{N}}\left(\frac{\mathrm{d}_{\mathrm{i}}^{\text {obs }}-\mathrm{d}_{\mathrm{i}}^{\text {pre }}}{\sigma_{\mathrm{i}}}\right)^{2},
$$

where $\mathrm{d}_{\mathrm{i}}^{\mathrm{obs}}, \mathrm{d}_{\mathrm{i}}^{\text {pre }}$, and $\sigma_{\mathrm{i}}$ are observation data, calculated data, and standard deviation from each datum, respectively. The model objective function $\left(\varphi_{\mathrm{m}}\right)$ is defined by Krahenbuhl [18] as:

$$
\begin{aligned}
& \varphi_{\mathrm{m}} \\
& =\alpha_{\mathrm{s}} \int_{\mathrm{V}}\left(\mathrm{w}(\mathrm{z})\left[\bar{\tau}-\overline{\tau_{0}}\right]\right)^{2} \mathrm{dv}+\alpha_{\mathrm{x}} \int_{\mathrm{V}}\left(\frac{\partial \mathrm{w}(\mathrm{z})\left[\bar{\tau}-\overline{\tau_{0}}\right]}{\partial \mathrm{x}}\right)^{2} \mathrm{dv} \\
& +\alpha_{\mathrm{y}} \int_{\mathrm{V}}\left(\frac{\partial \mathrm{w}(\mathrm{z})\left[\bar{\tau}-\overline{\tau_{0}}\right]}{\partial \mathrm{y}}\right)^{2} \mathrm{dv} \alpha_{\mathrm{z}} \int_{\mathrm{V}}\left(\frac{\partial \mathrm{w}(\mathrm{z})\left[\bar{\tau}-\overline{\tau_{0}}\right]}{\partial \mathrm{z}}\right)^{2} \mathrm{dv},
\end{aligned}
$$

where $\alpha_{\mathrm{s}}, \alpha_{\mathrm{x}}, \alpha_{\mathrm{y}}, \alpha_{\mathrm{z}}, \mathrm{V}, \tau$, and $\tau_{0}$ are, respectively: overall model relative weight, $\mathrm{X}$-axis relative weight, $\mathrm{Y}$-axis relative weight, $\mathrm{Z}$-axis relative weight, zone of reservoir model in the subsurface, alphabet model parameter, and reference model. In this paper, we set the values $\alpha_{s}=\alpha_{x}=\alpha_{y}=\alpha_{z}=1$. Distance weighting $(\mathrm{w}(\mathrm{z}))$ was defined as:

$$
w\left(\overline{z_{j}}\right)=\frac{1}{\sqrt{\Delta z_{j}}}\left\{\sum_{i=1}^{N}\left[\int_{\Delta V_{j}} \frac{d v}{\left(R+R_{0}\right)^{\beta}}\right]^{2}\right\}^{\frac{1}{4}} \text {, with } j=1, \ldots, M
$$

where $\Delta V$ is volume of the $\mathrm{j}^{\text {th }}$ cell, $R_{0}$ is a small constant, $R$ is distance between a point in $\Delta \mathrm{V}_{\mathrm{j}}$ and the $\mathrm{i}^{\text {th }}$ observation point. Exponent $\beta=2$, which is theoretically consistent with the fact that gravity fields decay as inverse distance squared. The general form of this weighting function is advantageous when applied to the inversion of data sets acquired in areas with a high topographic relief.

The objective function $\varphi$, as shown in Eq. (1), gives limitations to the possibility number of the model. Although the inverse problem is still non-unique, the possibility number is no longer 'infinite'. The need to achieve fitted or overfitted data can be reduced by minimization of the objective function. The model objective function $\left(\varphi_{\mathrm{m}}\right)$ narrows down 'infinite' models by considering what is geologically reasonable. Minimization of $\varphi_{\mathrm{m}}$ produces small size and minimum- 
structure complexity. Trade-off parameter $\lambda$ is assigned to control the balance between $\varphi_{\mathrm{d}}$ and $\varphi_{\mathrm{m}}$. As a final result, we expect the model to not be over-fitting with respect to noisy data acquisition.

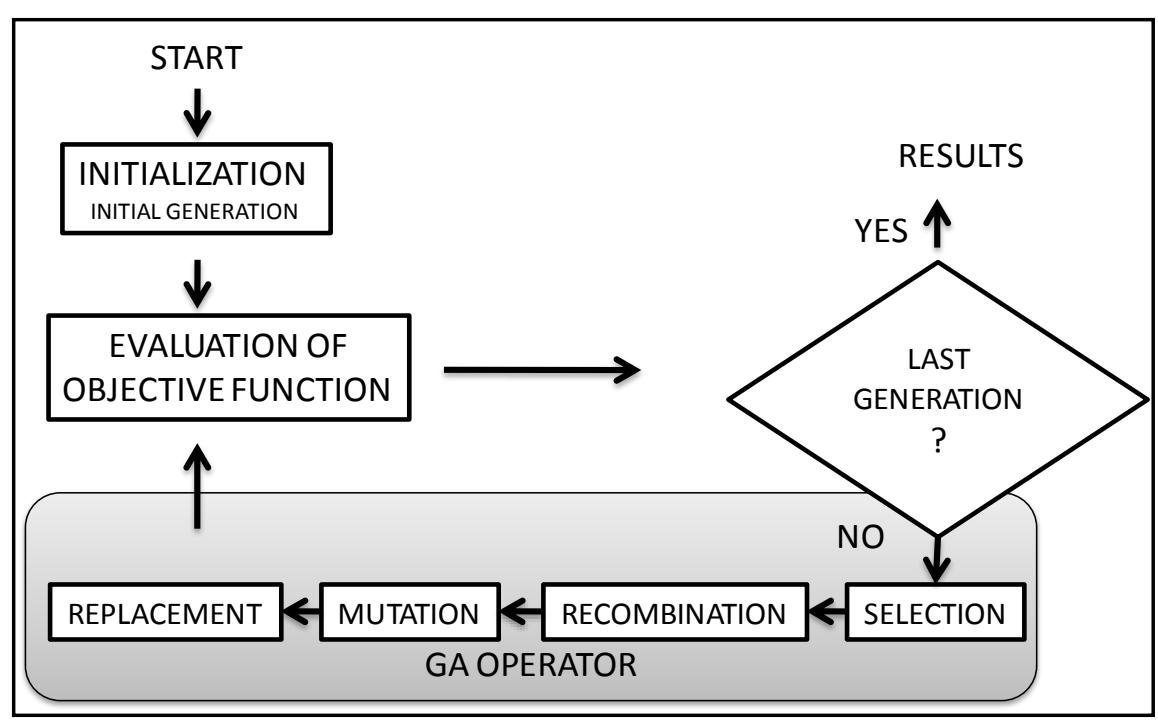

Figure 1 Flow-chart of GA (modified from [18]).

We chose the GA method as the optimization strategy to seek the inverse solution of this alphabet formulation. A guided random search technique such as a GA is derivative minimization free. A GA flowchart is shown in Figure 1. Generally, a GA works with an initial population consisting of random individuals. First, random individuals are generated as the initialization stage. Each individual represents a possible solution in the form of a set of chromosomes. A set of chromosomes is a combination of discrete model parameters A, B, and C. A perturbation model in GA terms involves artificial genetic operators (selection, recombination, mutation, and replacement). During the process of model exploration, the magnitude of the model parameters does not necessarily change in relation to the calculation. When a model parameter is selected for modification or recombination, the GA only changes that parameter into limited options of possible discrete values. In this study, the options of possible discrete values were A, B, or C. Forward calculation decided whether $\mathrm{A}, \mathrm{B}$, or $\mathrm{C}$ was most suitable as spatial model of the subsurface. The best solution was expected after calculating the last GA generation. 


\section{Synthetic Data}

The forward calculation of synthetic data uses an equation of gravitation caused by a 3-D polygonal prism body. The vertical component of gravitation in point $\mathrm{O}(0,0,0)$ caused by a body as shown in Figure 2, mathematically expressed with the following equation:

$$
g_{z}=G \rho \int_{z_{1}}^{z_{2}} \int_{y_{1}}^{y_{2}} \int_{x_{1}}^{x_{2}} \frac{z}{\left(x^{2}+y^{2}+z^{2}\right)^{3 / 2}} d x d y d z .
$$

Next, by integral Eq. (5) we get Eq. (6):

$$
g_{z}=\left.\left.\left.G \rho\left[\begin{array}{c}
x \ln (y+r)+ \\
y \ln (x+r)-z \tan ^{-1}\left(\frac{x y}{z r}\right)
\end{array}\right]\right|_{x_{1}} ^{x_{2}}\right|_{y_{1}} ^{y_{2}}\right|_{z_{1}} ^{z_{2}} .
$$

Eq. (6) is the gravitation effect of one prism body, while for the overall model, consisting of several prism bodies, the calculation is conducted cumulatively. Eq. (6) is described by Pluoff [19] numerically as Eq. (7):

$$
\begin{gathered}
\mathrm{g}_{\mathrm{z}}=\mathrm{G} \rho \sum_{\mathrm{i}=1}^{2} \sum_{\mathrm{i}=1}^{2} \sum_{\mathrm{i}=1}^{2} \mu_{\mathrm{ijk}}\left[\mathrm{z}_{\mathrm{k}} \tan ^{-1} \frac{\mathrm{x}_{\mathrm{i}} \mathrm{y}_{\mathrm{i}}}{\mathrm{z}_{\mathrm{k}} \mathrm{R}_{\mathrm{ijk}}}-\mathrm{x}_{\mathrm{i}} \log \left(\mathrm{R}_{\mathrm{ijk}}+\mathrm{x}_{\mathrm{i}}\right)\right. \\
\left.-\mathrm{y}_{\mathrm{i}} \log \left(\mathrm{R}_{\mathrm{ijk}}+\mathrm{y}_{\mathrm{i}}\right)\right],
\end{gathered}
$$

where: $r=\sqrt{x^{2}+y^{2}+z^{2}}, R_{i j k}=\sqrt{x_{i}^{2}+y_{j}^{2}+z_{k}^{2}}$, and $\mu_{i j k}=(-1)^{i}(-1)^{j}(-1)^{k}$.

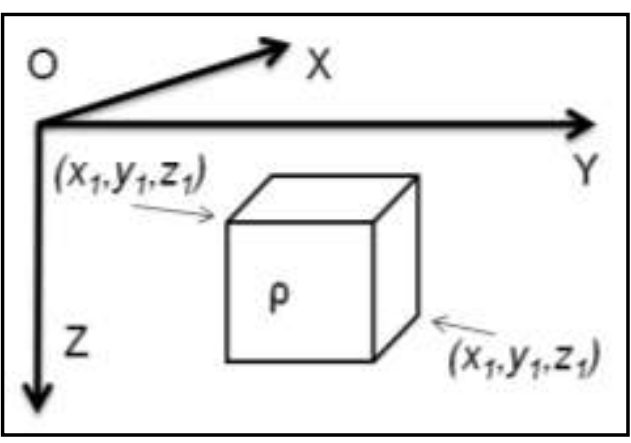

Figure 2 Illustration of 3-D prism with homogenous density ( $\rho$ ) and dimensions: $\mathrm{x}_{1} \leq \mathrm{x} \leq \mathrm{x}_{2}, \mathrm{y}_{1} \leq \mathrm{y} \leq \mathrm{y}_{2}, \mathrm{z}_{1} \leq \mathrm{z} \leq \mathrm{z}_{2}$. 


\subsection{Model A1}

Synthetic data were utilized for a single-inversion performance test calculated from model A1 (Figure 3). We show a single inversion using the GA for illustrative purposes. The time-lapse density values were distributed in a discrete-sized single reservoir with $(40 \times 40 \times 1)$ cells with uniform dimensions $\left(25 \times 25 \times 25 \mathrm{~m}^{3}\right)$. Positive and negative density changes were located in the NW and SE area respectively. As a numeric example, the model parameters $(\Delta \rho)$ to be reconstructed were $-0.15,0$, and $0.15 \mathrm{~g} / \mathrm{cc}$. The reservoir depth and topographic surface are shown in Figure 3(a).

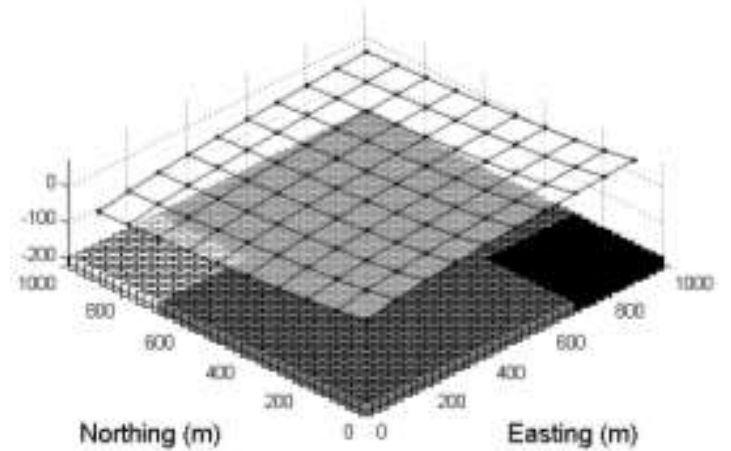

(a)

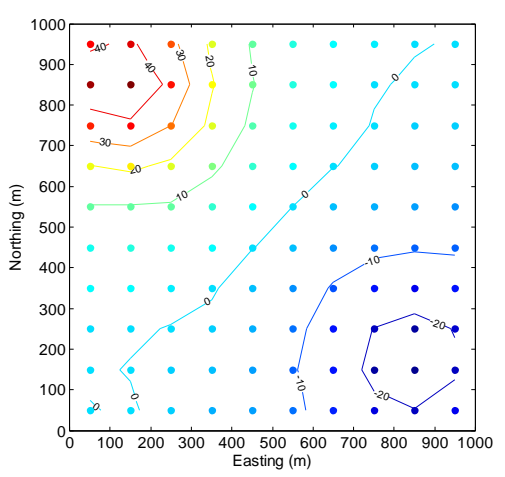

(b)

Figure 3 Model A1: (a) grid station positions on topographic surface over single-layer reservoir and (b) forward calculation with random noise [normal, 0 , 0.3 ] (contour in $\mu \mathrm{Gal}$ ).

The forward calculation of synthetic data was conducted over 100 stations distributed on the surface with a station grid interval of 100 meters in the $\mathrm{X}$-axis and $\mathrm{Y}$-axis direction. Random noise was added in the calculation with a normal distribution, zero mean, and standard deviation 0.3 (Figure 3(b)). Model A1 was used for illustrative purposes only, so we considered a relatively small level of noise, close to noise-free.

\subsection{Model A2}

Synthetic data were utilized for a GA operator performance test calculated from model A2 (Figure 4). This model is the same as the previous one, with a top reservoir depth of $200 \mathrm{~m}$ from a flat surface. Random noise was added in the calculation with normal distribution, zero mean, and standard deviation $5 \mu \mathrm{Gal}$ (Figure 5). 


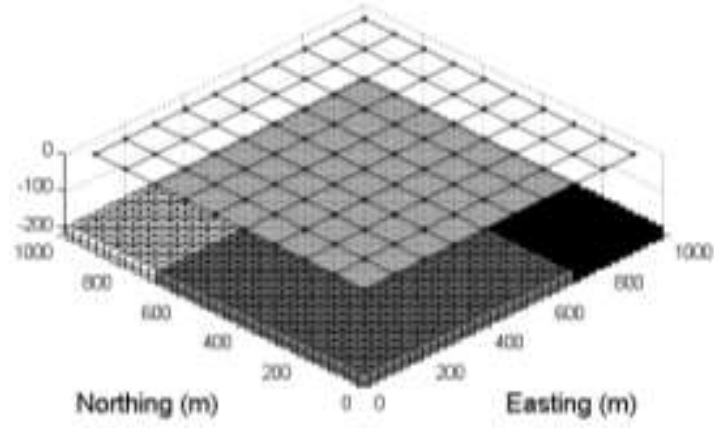

(a)

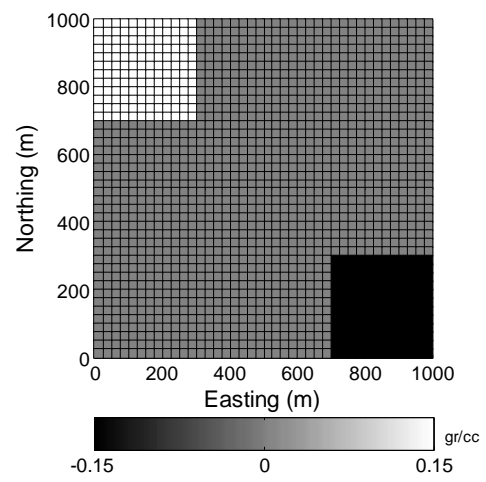

(b)

Figure 4 Model A2: (a) grid station positions on flat surface $(\mathrm{z}=0 \mathrm{~m})$ and (b) $\mathrm{X}-\mathrm{Y}$ view density distribution for model A2.

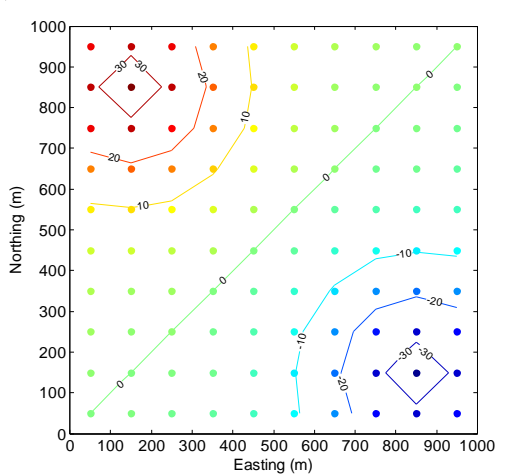

(a)

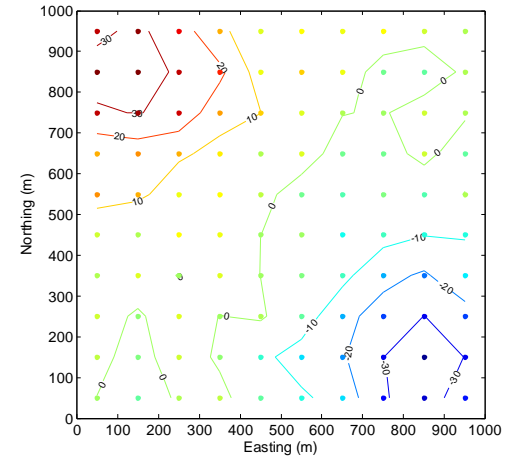

(b)

Figure 5 Forward calculation from model A2 (contour in $\mu \mathrm{Gal}$ ): (a) noise-free and (b) synthetic data with random-noise [normal, 0, 5].

\subsection{Model B}

Model B illustrates the activities in an oil field with 4 injection wells and 9 production wells. Pore-fluid density changes for model B utilized the assumption of several parameter values $(0.8 \mathrm{~g} / \mathrm{cc}$ for oil density, $1.0 \mathrm{~g} / \mathrm{cc}$ for water density and $30 \%$ for porosity). Bulk densities, before $(i=1)$ and after ( $i=$ 2) the time-lapse period, could be calculated using Eq. (8) from Schön [20]:

$$
\rho_{\mathrm{i}}=(1-\phi) \rho_{\mathrm{m}}+\phi \rho_{\mathrm{f}}, \text { with } \mathrm{i}=1,2
$$


where $\rho, \rho_{\mathrm{m}}, \rho_{\mathrm{f}}, \rho_{\mathrm{m}}$, and $\phi$ are bulk density, solid matrix material density, porefluid density, and total porosity, respectively. After the density change $(\Delta \rho)$ was calculated by Eq. (9), we got $0.06 \mathrm{~g} / \mathrm{cc}$ and $-0.24 \mathrm{~g} / \mathrm{cc}$ for the injection and production scenario, respectively.

$$
\Delta \rho=\rho_{2}-\rho_{1} .
$$

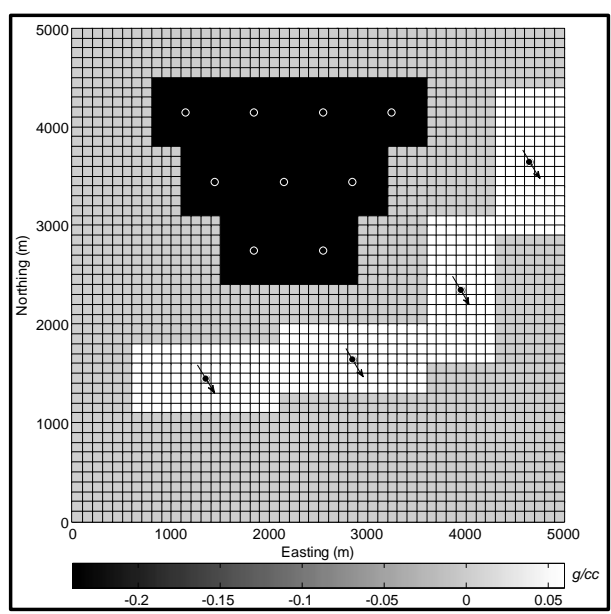

Figure 6 Model B.

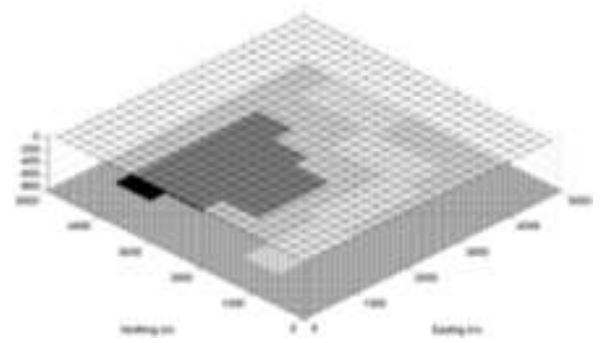

(a)

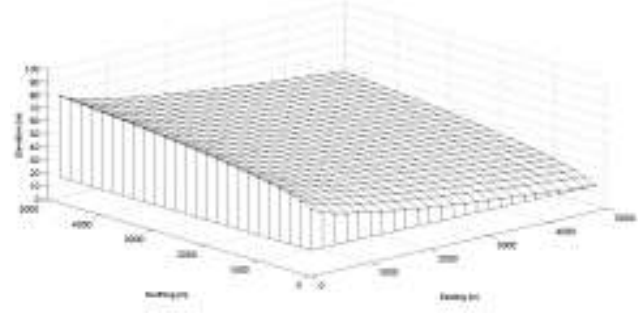

(b)

Figure 7 Illustration of (a) grid station positions on topographic surface on top of model B, and (b) zoom in on topographic variation on top of model B.

Compared to the previous models (A1 and A2), model B (Figure 6) has a larger matrix size. We present the performance of the GA inversion for a larger matrix, because when dealing with real data the matrix will be at least this size. Time-lapse density values were distributed in a discrete-sized single-layer reservoir with $(50 \times 50 \times 1)$ cells of uniform dimensions $\left(100 \times 100 \times 30 \mathrm{~m}^{3}\right)$. The reservoir was located at a depth of $800 \mathrm{~m}$ below zero MSL (Figure 7(a)). The 625 surface stations were arranged as an ideal grid with a $200 \mathrm{~m}$ interval 
distributed on the topographic surface, as shown in Figure 7(b). Random noise was added in the forward calculation with normal distribution, zero mean, and standard deviation 5 (Figure 8).

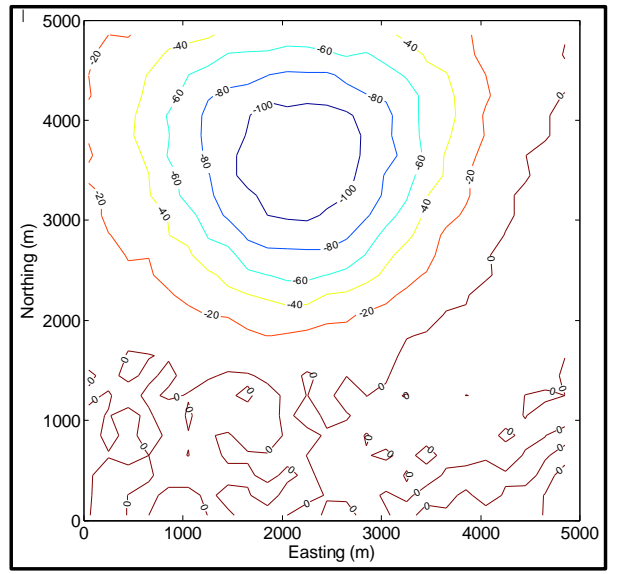

Figure 8 Synthetic data B with random-noise [normal, 0, 5] (contours in $\mu \mathrm{Gal}$ ).

\section{$4 \quad$ Inversion Performance}

\subsection{Single-Inversion Performance Model A1}

In this section, we describe the single inversion using the GA in order to show the simple steps of the algorithm, for illustrative purposes. GA inversion of synthetic data from model A1 was conducted to resolve the density distribution in $(40 \times 40 \times 1)$ cells of the reservoir model. During the initialization stage, the program generates 5 individuals. The chromosomes for each individual are arranged from random discrete model parameters (A, B, and $\mathrm{C}$ ). The termination criterion used for this performance test was 30 generations for every performance sample.

The data misfit of the single inversion shown in Figure 9(a) resulted with a specification of $2.00 \mathrm{~GB}$ RAM and $3.30 \mathrm{GHz}$ CPU. The elapsed time after calculating an entire generation number (30) was 5.814 seconds. In the last generation, the best individual produced the minimum value of the objective function (472.654, as shown in Figure 9(b)).

The best-individual model resulted from the single-inversion GA (as shown in Figure 10) gave a sharp characterization. The algorithm was capable of identifying positive and negative density changes in the NW and SE part of the reservoir model respectively. Although the true cell recovery (TCR) of the 
inversion model was relatively high $(97.750 \%)$, this numerical example still shows disturbing features near the reference zone boundaries.

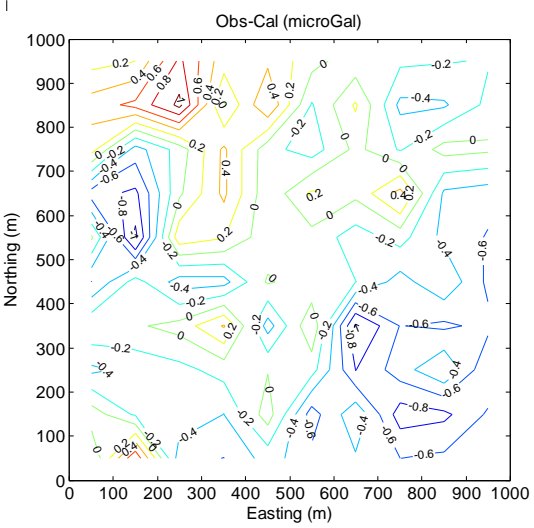

(a)

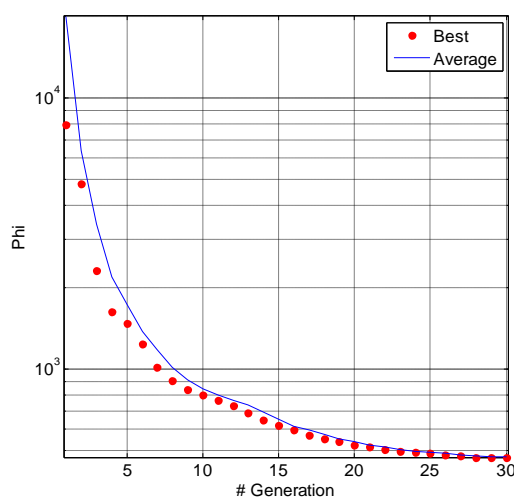

(b)

Figure 9 Single-inversion performance of synthetic data A1: (a) data misfit and (b) objective function vs generation number.

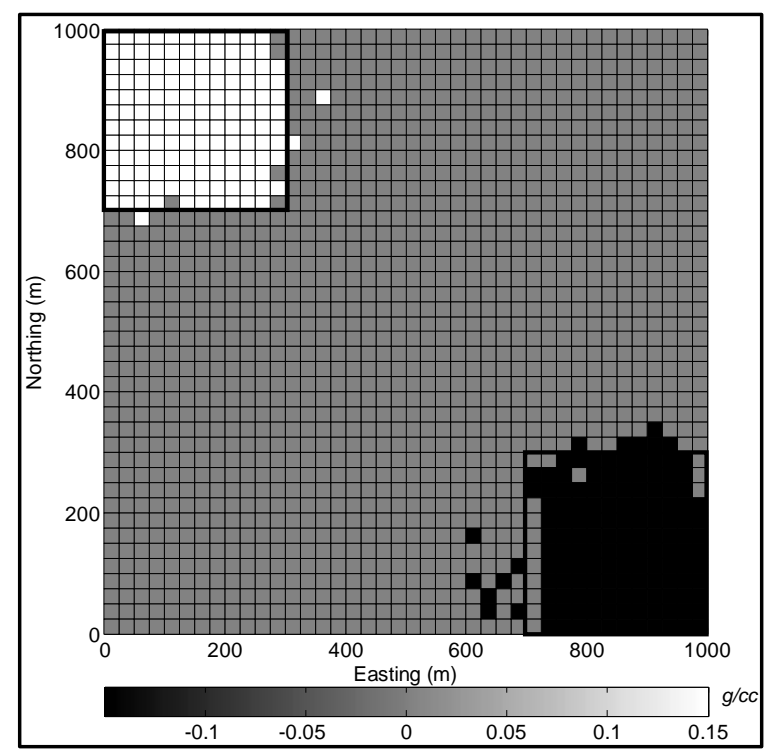

Figure 10 Best individual resulted from single-inversion GA with synthetic data model A1 (thick black lines indicate boundaries of density changes from synthetic model). 


\subsection{GA Operator Performance Test Model A2}

Based on the flowchart shown in Figure 1, the GA operators had significant impact, proving their effectiveness in model parameter exploration. Because of the rapid growth of GA adaptations for optimization tools, there are several variations of GA operators that can be combined in inversion program designs. Several variations of the GA operators tested in this study can be found in the literature [21-27]. The performance test combined only certain/selected GA operators, because not all GA operators (as mentioned in the references) are suitable to be applied in our formulation. Some of the described floating-point operator mechanisms are difficult to adapt to our formulation.

Every sample resulted from the same treatment (inversion set-up 10, individuals and last generation both 5000). In order to draw conclusions from this test, considering that the GA works with random numbers, every GA operator combination tested was summarized by the average value of the 50 performance samples (as shown in Table 1).

Table 1 Comparison of GA operator combinations on the basis of average value of 50 performance samples.

\begin{tabular}{|c|c|c|c|c|c|c|}
\hline Design & GA Operator & $\begin{array}{c}5000 \text { Gen } \\
\text { ET (s) }\end{array}$ & $\begin{array}{c}\text { Best } \\
\text { Phi }\end{array}$ & TCR & $\begin{array}{l}\text { Conv } \\
\text { (\#Gen) }\end{array}$ & $\begin{array}{l}\text { Conv } \\
\text { ET (s) }\end{array}$ \\
\hline 1 & RWS-MPCO-HOF-ESR & 29.930 & 1238.79 & 95.91 & 4549.74 & 27.234 \\
\hline 2 & TS-MPCO-HOF-ESR & 30.120 & 1238.87 & 95.89 & 4487.22 & 27.031 \\
\hline 3 & SUS-MPCO-HOF-ESR & 29.468 & 1238.39 & 95.95 & 4441.66 & 26.177 \\
\hline 4 & SUS-DPCO-HOF-ESR & 27.078 & 1239.08 & 95.94 & 4729.36 & 25.613 \\
\hline 5 & SUS-SPCO-HOF-ESR & 26.144 & 1241.58 & 95.83 & 4915.78 & 25.703 \\
\hline 6 & $\begin{array}{l}\text { SUS-MPCO- } \\
\text { QSA/100G-ESR }\end{array}$ & 35.805 & 1239.16 & 95.97 & 2976.72 & 21.316 \\
\hline 7 & $\begin{array}{l}\text { SUS-MPCO-QSA/G- } \\
\text { ESR }\end{array}$ & 965.596 & 1237.70 & 96.02 & 129.80 & 25.067 \\
\hline 8 & $\begin{array}{l}\text { SUS-MPCO-QSA/G- } \\
\text { SSR }\end{array}$ & 905.214 & 1237.61 & 96.03 & 129.18 & 23.387 \\
\hline 9 & SUS-MPCO-QSA/G-ND & 868.999 & 1237.09 & 96.04 & 121.78 & 21.165 \\
\hline
\end{tabular}

\subsubsection{Selection Operator Test}

The individual selection stage in the GA decides parent pairs based on a fitness value. Potential solution models with less data misfit have a higher probability to be selected. The characteristics of the individuals with a higher fitness have a higher probability to survive in the next generation.

The three selection operators tested were: SUS (stochastic universal sampling), RWS (roullete wheel selection with fitness-proportional probability), and TS 
(tournament selection). In order to make a comparison, each selection operator was combined with the same GA operators (recombination, mutation, and replacement, i.e. MPCO (multi-point crossover), HOF (half offspring flips), and ESR (evolution strategy replacement) respectively). Based on the comparison of the selection operator tests (design \#1, \#2, and \#3) as shown in Table 1 and Figure 11, we chose SUS as the most promising selection operator mechanism for this inversion. The average values of the 50 SUS performance samples can be summarized as follows: 26.177 seconds ET and 4441.66 as generation number of convergence. SUS performance showed the smallest Phi (objective function) and the highest TCR compared to RWS and TS.

\subsubsection{Recombination Operator Test}

Recombination in the GA combines the chromosomes of selected parent pairs. The recombination process introduces offspring (hopefully as fresh candidates) for new potential solutions. In terms of model parameter exploration, recombination represents teamwork to reach optimization without the slow process of perturbation.

The three recombination operators tested were: MPCO (multi-point crossover), SPCO (single-point crossover), and DBCO (double-point crossover). In order to make a comparison, each recombination operator was combined with the same GA operators (selection, mutation, and replacement, i.e. SUS, HOF, and ESR respectively). Based on the comparison of the recombination operator tests (design \#3, \#4, and \#5) as shown in Table 1 and Figure 11, MPCO (with 10point crossover) was a promising recombination operator mechanism for this inversion. The average values of the 50 MPCO performance samples can be summarized as follows: 26.177 seconds ET and 4441.66 as generation number of convergence. MPCO performance showed the smallest Phi (objective function) and the highest TCR compared to DPCO and SPCO.

\subsubsection{Mutation Operator Test}

Mutation in the GA provides an important rule to introduce new characteristics of chromosomes in order to prevent premature convergence. The three mutation operators tested were: HOF (half offspring flips), QSA/100G (quenched simulated annealing per $100^{\text {th }}$ generation), and QSA/G (quenched simulated annealing per generation). In order to make a comparison, each mutation operator was combined with the same GA operators (selection, recombination, and replacement, i.e. SUS, MPCO, and ESR, respectively).

HOF in the mutation stage works in half the number of offspring for every generation. The mechanism of HOF is as follows: the algorithm will randomly choose a single chromosome and then flip it into another discrete model 
parameter. For example, when the randomly selected single chromosome is A, then HOF will flip it into B or C. An analog mechanism is applied to the other two possibilities for B or C. In this study, HOF was applied continuously in every generation.

QSA/100G is an adaptation of the hybrid optimization of Krahenbuhl [18], but in this study it was used in the mutation stage. Krahenbuhl [18] describes QSA as a simple form of simulated annealing without temperature cooling criterion. Here, we describe the mechanism to adapt the alphabet formulation. The flipping mechanism is similar to that of HOF, but this operator functions as a local search in the top individuals of the population. After the flipping process, the objective function of the new model is evaluated directly. QSA only accepts lateral and downhill movement of the objective function. The term '/100G' indicates that QSA performs every 100 generations. As shown in Figure 11, the performance test showed significant efficiency, especially for every QSA performed the ladder-shaped curve has a downward slope.

Most GA designs use a probabilistic method to perform mutation, but we chose to apply significant QSA continuously in every generation (QSA/G). Such kind of mechanism is needed in this GA inversion in order to speed up model parameter exploration and reduce the number of generations.

Based on the comparison of the mutation operator tests (\#3, \#6, and \#7) as shown in Table 1 and Figure 11, QSA/G is the most promising mutation operator mechanism for this inversion. The average values of the $50 \mathrm{QSA} / \mathrm{G}$ performance samples can be summarized as follows: 25.067 seconds ET and 129.80 as generation number of convergence. QSA/G performance showed the smallest Phi (objective function) and the highest TCR compared to QSA/100G and $\mathrm{HOF}$.

\subsubsection{Replacement Operator Test}

The replacement stage in GA decides about continuity from generation to generation. The three replacement operators tested were: ESR (evolution strategy replacement), SSR (steady state replacement), and ND (no duplicity). In order to make a comparison, each replacement operator was combined with the same GA operators (selection, recombination, and mutation, i.e. SUS, MPCO, and QSA/G, respectively).

ESR is replacement with evolution schematics involving parents and offspring competing for existence in the next generation. SSR is replacement by replacing all parents with offspring. These kinds of ESR and SSR have the possibility to produce duplicate/uniform individuals as a monopoly of the best individual. ND 
as replacement operator proposes an alternative schematic filter tool for nextgeneration members. ND does not allow any duplication of individuals into the next generation. This kind of schematic maintains the heterogeneity of the chromosomes among the population members.

Based on the comparison of the replacement operator tests (\#7, \#8, and \#9) as shown in Table 1 and Figure 11, we chose ND as the most promising replacement operator mechanism for this inversion. The average values of the $50 \mathrm{ND}$ samples can be summarized as follows: 21.165 seconds ET and 121.78 as generation number of convergence. ND performance showed the smallest Phi (objective function) and the highest TCR compared to ESR and SSR.

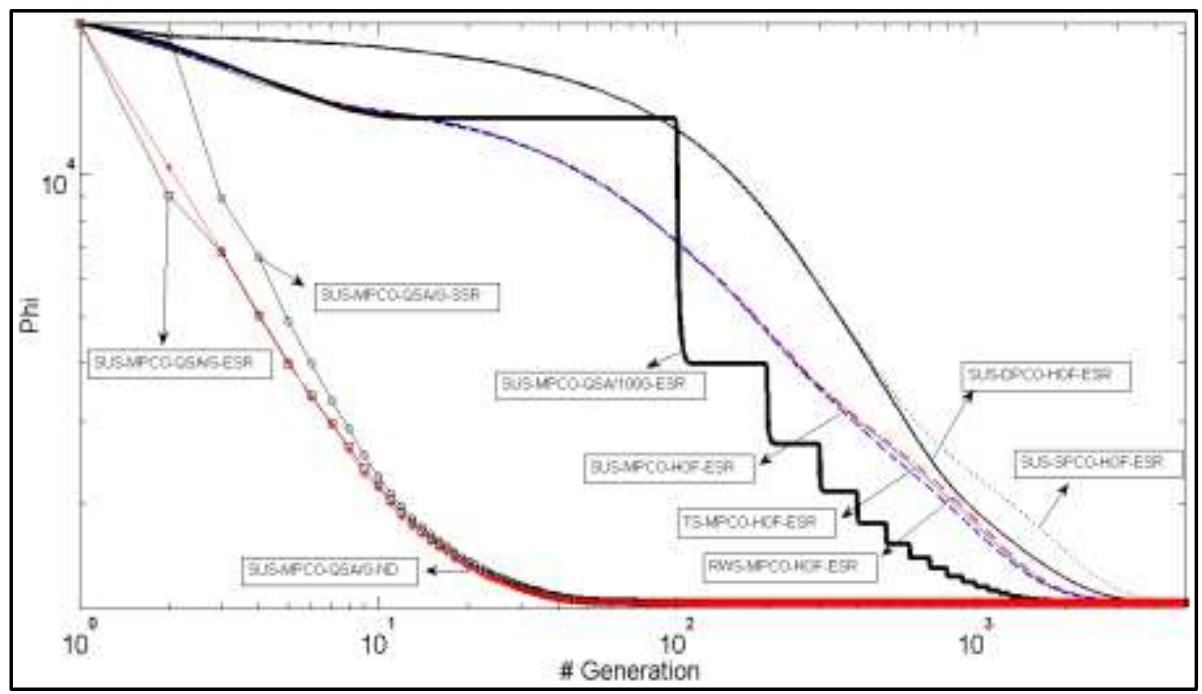

Figure 11 Comparison of 9 combinations of GA operator performance.

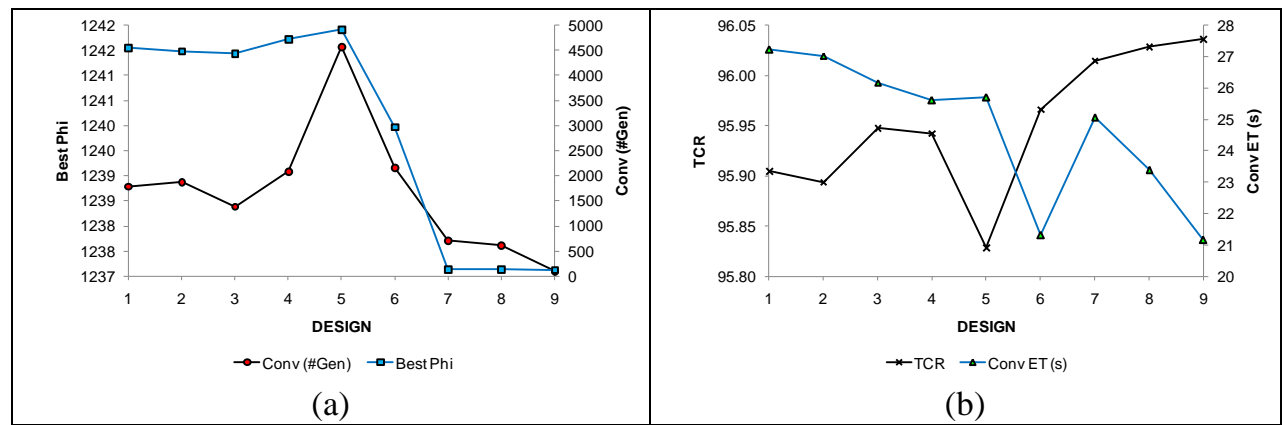

Figure 12 Comparison of: (a) average best Phi with average generation number for model convergence, and (b) average percentage of true cell recovery with average elapsed time for each single inversion. 
We looked for the most efficient mechanism regarding computational time, but we also looked for inverse results that were close to the synthetic model (TCR close to 100\%) and followed the mechanism's performance in minimizing the objective function (smallest Phi). Comparison of average best Phi with average generation number (Figure 12(a)) shows that design \# 9 had minimum value. The average percentage of true cell recovery for design \#9 was the highest with the shortest average ET for each single inversion (Figure 12(b)). The complete performance test, as shown in Table 1 and the curve comparison (Figure 11) with 50 samples, shows that the combination of SUS-MPCO-QSA/G-ND delivered the most promising results.

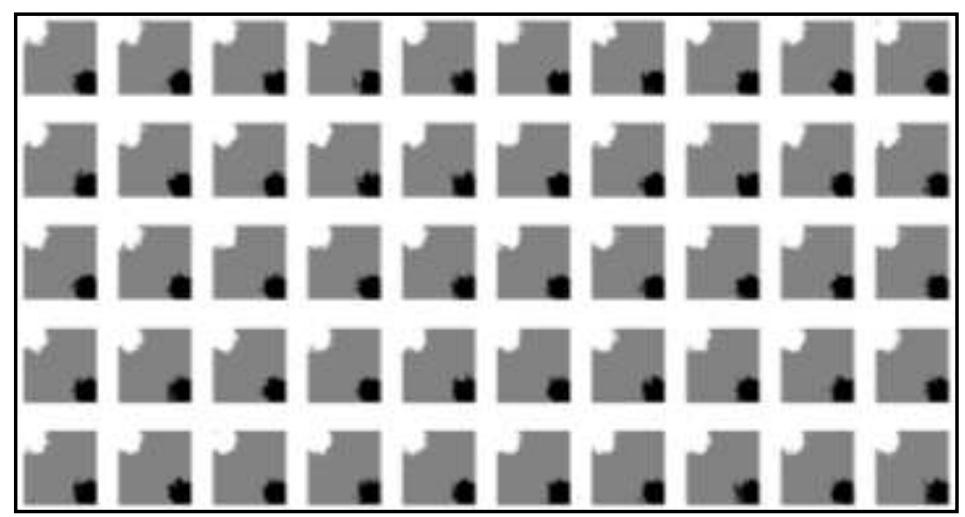

Figure 13 Best individuals from 50 GA multiple-inversion samples.

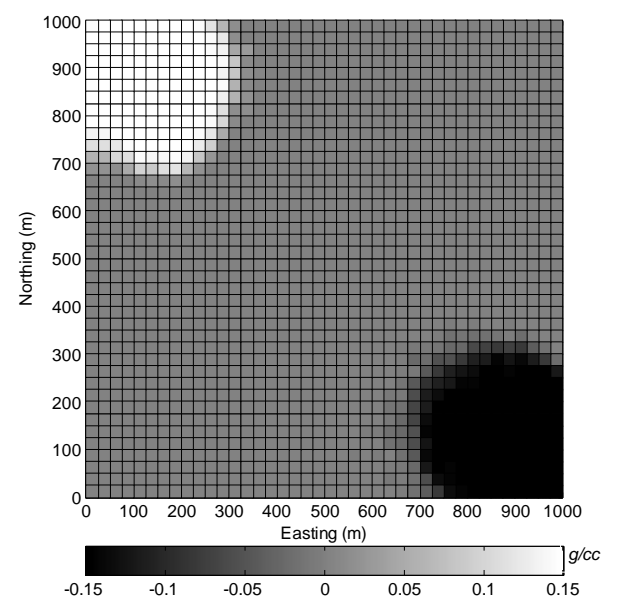

(a)

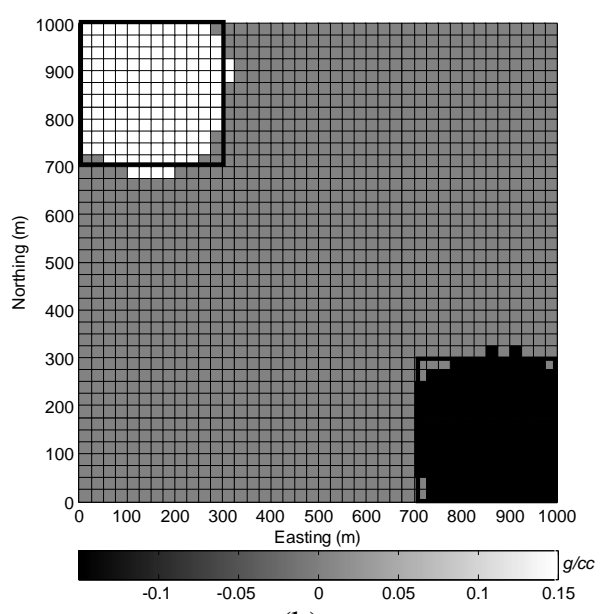

(b)

Figure 14 GA inversion results of model A2: (a) averaged model from 50 samples and (b) clustering model with interpretation of $\mathrm{A}>0.075 \mathrm{~g} / \mathrm{cc}$ and $\mathrm{C}<-$ $0.075 \mathrm{~g} / \mathrm{cc}$ (thick black lines indicate boundaries of density changes from synthetic model). 
As an example, we used 50 samples of the GA multiple-inversion (Figure 13) that resulted after 913.270 seconds ET using the same PC. We applied the clustering interpretation of the averaged model as shown in Figure 14(a). The clustering interpretation produced 98.625\% TCR, as shown in Figure 14(b).

GA generally defines the best individual at the end of a generation as the final solution, although every time the GA was performed, it 'never' seemed to provide a unique best individual. As shown in Figure 13, the best individuals from the $50 \mathrm{GA}$ samples always differed from one another. Qualitatively, every best solution resulted from the $50 \mathrm{GA}$ samples showed the capability to locate positive and negative density changes, respectively in the NW and SE part of the reservoir model. The solution for sharp characterization of the density change boundaries can be optimized by calculating the averaged model as Krahenbuhl, et al. [28]. A clustering interpretation of the average model set needs to be carried out. Multiple-inversion conducted this way was considered in order to push down disturbing features near the reference zone boundaries. Hopefully, this would lead to a quantitative improvement regarding the density change boundaries.

\subsection{Multiple-Inversion Performance Model B}

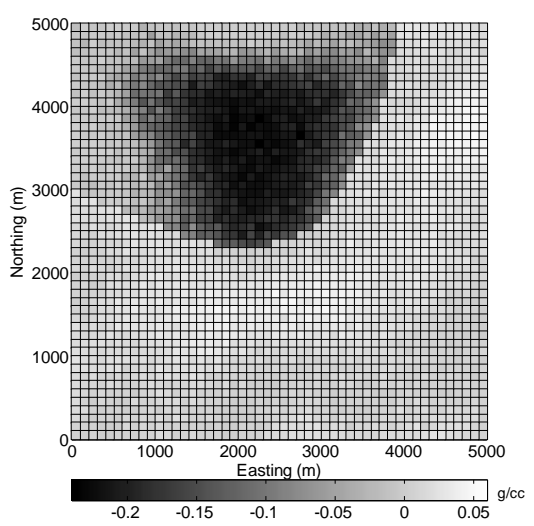

(a)

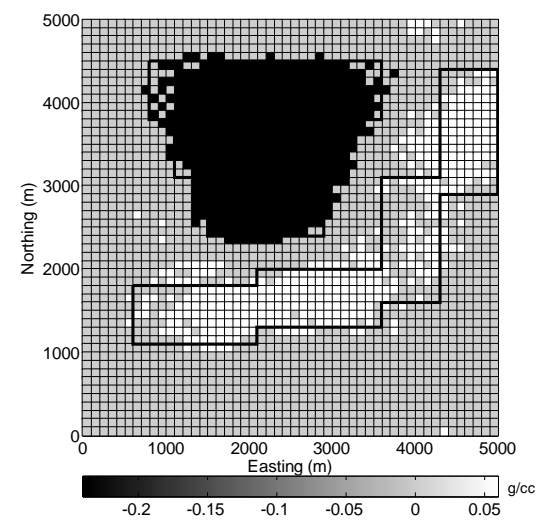

(b)

Figure 15 GA inversion results for model B: (a) average model from 50 samples and (b) clustering model with interpretation of $\mathrm{A}>0.0003 \mathrm{~g} / \mathrm{cc}$ and $\mathrm{C}<$ $-0.0012 \mathrm{~g} / \mathrm{cc}$ (thick black lines indicate density change boundaries from synthetic model).

After having defined the best combination of GA operators in view of singleinversion performance and looking at the way to optimize the GA inversion results for a simple model, we tried out the GA using synthetic data calculated from model B. The inversion parameters were set up using 50 as value for both 
the individuals and the last generation. We took $50 \mathrm{GA}$ multiple-inversion samples that resulted after 10407.849 seconds ET. We applied a clustering interpretation of the averaged model as shown in Figure 15(a). The clustering interpretation produced $88.160 \%$ TCR, as shown in Figure 15(b).

\section{Conclusion}

We have developed an alphabet formulation for inverting time-lapse microgravity data for single-layer reservoirs. Inverse modeling with alphabet formulation is capable of providing solutions for characterizing positive density change $(+\Delta \rho)$ and negative density change $(-\Delta \rho)$ with respect to a reference value $(0 \mathrm{gr} / \mathrm{cc})$. Our initial tests with synthetic data showed that the alphabet formulation provided an effective means to incorporate interpreted density changes while maintaining the flexibility of the density inversion.

We have explored combinations of GA operators to inverse time-lapse microgravity using a synthetic single-reservoir model. Analysis of several samples showed that the combination of SUS-MPCO-QSA/G-ND had the most promising results. Practical application to larger and deeper reservoir models was shown with Model B. A quantitative solution with a higher confidence level and sharp boundaries of density change conducted by a clustering interpretation of the averaged model set resulted from multiple inversion. Currently, we are using real data to explore multiple QSA inversion alone as optimization strategy to seek the inverse solution. Hopefully, calculations involving more complex reservoir parameters (such as: reservoir thickness and porosity variation) can be useful to support reservoir simulation research.

\section{References}

[1] Kadir, W.G.A., Santoso, D. \& Sarkowi, M., Mathematical Model of Time-Lapse Vertical Gradient Microgravity Measurement and Its Application for Subsurface Mass Change and Vertical Ground Movement (Subsidence) Identification, Case Study: Semarang Alluvial Plain, Central Java, Indonesia, Far East Journal Mathematical Sciences, 37(2), pp. 181-192, 2010.

[2] Santoso, D., Kadir, W.G.A., Alawiyah, S., Setianingsih, Wahyudi, E.J., Sarkowi, M. \& Minardi, S., Understanding the Time-Lapse Microgravity Response due to Subsidence and Groundwater Level Lowering, The Contribution of Geosciences to Human Security, Logos Verlag Berlin, pp. 27-48, 2011.

[3] Li, Y. \& Oldenburg, D.W., 3-D Inversion of Gravity Data, Geophysics, 63(1), pp. 109-119, 1998. 
[4] Krahenbuhl, R.A. \& Li, Y., Gravity Inversion using a Binary Formulation, SEG International Exposition and $72^{\text {nd }}$ Annual Meeting, Salt Lake City, Utah, by Society of Exploration Geophysicists, pp. 755$758,2002$.

[5] Krahenbuhl, R.A. \& Li, Y., Hybrid Optimization for a Binary Inverse Problem, SEG International Exposition and 74th Annual Meeting, Denver, Colorado, by Society of Exploration Geophysicists, pp. 782-785, 2004.

[6] Krahenbuhl, R.A. \& Li, Y., Inversion of Gravity Data Using a Binary Formulation, Geophysical Journal International, 167(2), pp. 543-556, 2006.

[7] Krahenbuhl, R.A. \& Li, Y., Time-lapse Gravity: A Numerical Demonstration Using Robust Inversion and Joint Interpretation of $4 D$ Surface and Borehole Data, Geophysics, 77(2), pp. G33-G43, 2012.

[8] Fujimitsu, Y., Nishijima, J., Shimosako, N., Ehara, S. \& Ikeda, K., Reservoir Monitoring by Repeat Gravity Measurements at the Takigami Geothermal Field, Central Kyushu, Japan, World Geothermal Congress, Beppu-Marioka, Japan, by International Geothermal Association, 2000.

[9] Nordquist, G.A., Acuna, J. \& Stimac, J., Precission Gravity Modeling and Interpretation at the Salak Geothermal Field, Indonesia, World Geothermal Congress, Bali, Indonesia, by International Geothermal Association, 2010.

[10] Kadir, W.G.A., Santoso, D., Alawiyah, S., Setianingsih, Widianto, E. Sardjito \& Waluyo, Microgravity Monitoring for Sandstone and Carbonate Reservoirs along Water Injection Activity-Case Study of Talang Jimar and Tambun Oil Fields, Indonesia, The Contribution of Geosciences to Human Security, Logos Verlag Berlin, pp. 127-140, 2011.

[11] Sarkowi, M., Budiarto, I. \& Atmojo, J.P., 4D Microgravity for Monitoring Coal Bed Methane Production Process: Case Study Rambutan Field (text in Indonesian), Proceedings of the Joint Convention Makasar HAGI-IAGI, Makasar, South Sulawesi, by Himpunan Ahli Geofisika Indonesia-Ikatan Ahli Geologi Indonesia, 2011.

[12] Setianingsih, Wahyudi, E.J., Kadir, W.G.A., Alawiyah, S., Wicaksono, R.A. \& Kusumah, Y.I., Case Study of Time-Lapse Microgravity Data on The Wayang Windu Geothermal Field, Indonesia: Quality Control of Data Acquisition and Data Application for Reservoir Analysis, SEGJ International Symposium, Kyoto, Japan, by by Society of Exploration Geophysics of Japan, 2011.

[13] Wahyudi, E.J., Inversion of Surface and Borehole Gravity Data Using Genetic Algorithm to Characterize Fluid Contact Movement (Text in Indonesian), Bandung, Indonesia, Institut Teknologi Bandung, 2009.

[14] Wahyudi, E.J., 2 Bit Inversion of $4 D$ Gravity Data Using Genetic Algorithm to Characterize Injection and Production Zone in Permeable 
Layer, Proceedings of the Joint Convention Makasar HAGI-IAGI, Makasar, South Sulawesi, by Himpunan Ahli Geofisika Indonesia-Ikatan Ahli Geologi Indonesia, 2011.

[15] Wahyudi, E.J., Kadir, W.G.A. \& Grandis, H., Inversion of Surface and Borehole Gravity Data Using Genetic Algorithm to Characterize Fluid Contact Movement (Text in Indonesian), Jurnal Geofisika, 2, 2009.

[16] Wahyudi, E.J., Kadir, W.G.A. \& Grandis, H., Inversion of Surface and Borehole Gravity Data Using Genetic Algorithm to Characterize Fluid Contact Movement, Bali 2010 International Geosciences Conference \& Exposition, Bali, Indonesia, by Himpunan Ahli Geofisika Indonesia, 2010.

[17] Wahyudi, E.J., Kadir, W.G.A. \& Grandis, H., 2 Bit Inversion of $4 D$ Gravity Data Using Genetic Algorithm to Characterize Injection and Production Zone in Permeable Layer, EGU General Assembly, Vienna, Austria, by European Geosciences Union, 2011.

[18] Krahenbuhl, R.A., Binary Inversion of Gravity Data for Salt Imaging, Colorado School of Mines, Golden, Colorado, USA, 2005.

[19] Plouff, D., Gravity and Magnetic Fields of Polygonal Prisms and Application to Magnetic Terrain Corrections, Geophysics, 41(4), pp. 727-741, 1976.

[20] Schön, J.H., Physical Properties of Rocks, Pergamon, Trowbridge, England, 1996.

[21] Blickle, T. \& Thiele, L., A Comparison of Selection Schemes Used in Genetic Algorithms, TIK-report, Zurich, Switzerland 1995.

[22] Sen, M. \& Stoffa, P.L., Global Optimization Methods in Geophysical Inversion, Elsevier, Amsterdam, Netherlands, 1995.

[23] Townsend, A.A.R., Genetic Algorithm-A Tutorial, http://citeseerx.ist. psu.edu/viewdoc/download?doi=10.1.1.184.3148\&rep=rep1\&type=pdf, 2003 (10 February 2013 ).

[24] Xu, H., Comparison of Genetic Operators on a General Genetic Algorithm Package, Oklahoma State University, Stillwater, Oklahoma, USA, 2005.

[25] Reeves, C., Handbook of Metaheuristics-Chapter 3 Genetic Algorithms, pp. 55-82, Kluwer Academic Publishers, Dordrecht, Netherlands, 2003.

[26] Sastry, K., Goldberg, D. \& Kendall, G., Search Methodologies-Chapter 4 Genetic Algorithms, Springer, New York, USA, 2005.

[27] Sivaraj, R. \& Ravichandran, T., A Review of Selection Methods in Genetic Algorithm, International Journal of Engineering Science and Technology, 3(5), pp. 3792-3797, 2011.

[28] Krahenbuhl, R.A., Li, Y. \& Davis, T., 4D Gravity Monitoring of Fluid Movement at Delhi Field, LA: A Feasibility Study with Seismic and Well Data, SEG International Exposition and 80th Annual Meeting, Denver, Utah, by Society of Exploration Geophysicists, 2010. 